\title{
Lagrangian tools to monitor transport and mixing in the ocean
}

\author{
S. V. Prants*, M. V. Budyansky and M. Yu. Uleysky \\ Pacific Oceanological Institute of the Russian Academy of Sciences, \\ Vladivostok, 690041, Russia
}

\begin{abstract}
We apply the Lagrangian approach to study surface transport and mixing in the ocean. New tools have been developed to track the motion of water masses, their origin and fate and to quantify transport and mixing. To illustrate the methods used we compute the Lagrangian synoptic maps a comparatively small marine bay, the Peter the Great Bay in the Japan Sea near Vladivostok city (Russia), and in a comparatively large region in the North Pacific, the Kuroshio Extension system. In the first case we use velocity data from a Japan Sea circulation numerical model and in the second one the velocity data are derived from satellite altimeter measurements of anomalies of the sea height distributed by AVISO.
\end{abstract}

Keywords: Mixing; Eddy; Lagrangian synoptic map; Marine bay; Kuroshio Extension.

\section{Introduction}

The ocean presents a variety of dynamical phenomena with different space scales ranging from millemeters to a few thousand of kilometers. Despite of that, large-scale coherent structures are easlily visible, say, at satellite images of the sea color and surface temperature and can be identified by means of

*E-mail: prants@poi.dvo.ru, http://dynalab.poi.dvo.ru 
in-situ measurements. The striking examples are the major western boundary oceanic currents, the Gulf Stream in the Atlantic and the Kuroshio in the Pacific. They are "rivers" with the warm water in the ocean with the width on the order of 100-200 km and the maximal speed of current at the surface of $2 \mathrm{~m} / \mathrm{s}$. Such currents separate waters with different physical, chemical and biological characteristics. The other examples are mesoscale (with the size of a few hundred of kilometers) and submesoscale (a few tenth of kilometers) eddies that can transport water over hundreds and even thousands of kilometers and can survive for months before breaking down. Being coherent features, they do not contain the same waters but exchange them with the surrounding ocean, the process known as mixing.

Lagrangian and dynamical systems methods have been developed to study large-scale transport and mixing in the ocean [4, 5, 3, 2, 6, 7, 12, 11]. The main purposes of those studies are to track the fluid motion, to elucidate and quantify transport and mixing processes. Simply speaking, we would like to know where these or those waters come from, what is their fate and how they mix in this or that region. In the Lagrangian approach one integrates trajectories for a large number of synthetic particles advected by an Eulerian velocity field

$$
\frac{d \vec{r}}{d t}=\vec{v}(\vec{r}, t) .
$$

The velocity field, $\vec{v}(\vec{r}, t)$, is supposed to be known analytically, numerically or estimated from satellite altimetry. While in the Eulerian approach we get frozen snapshots of data, Lagrangian diagnostics enable to quantify spatiotime variability of the velocity field. It has been established theoretically and experimentally that even a simple deterministic velocity field may cause practically unpredictable particle trajectories, the phenomenon known as chaotic advection [8, 5]. The real oceanic flows are not, of course, deterministic and regular, but if the Eulerian correlation time is large as compared to the Lagrangian one, the problem may be treated in the framework of chaotic advection concept.

It is important to separate chaotic and turbulent mixing in the ocean. The process of chaotic advection provides transport and mixing with the characteristic scales on the order of a few tenths or even hundreds of kilometers, whereas turbulence works at smaller scales. At a comparatively large scale, turbulent mixing is homogeneous whereas the chaotic one is not. Typical patterns of chaotic advection consist of large-scale convoluted curves visible in some surface-temperature and color satellite images. The effect 
of turbulent mixing is in small-scale fluctuations superimposed on the largescale convoluted curves. If the velocity field on comparatively large scales is quasicoherent in space and quasiregular in time but the motion of tracers is mainly irregular, one deals with chaotic mixing. Turbulent mixing means that the velocity field is irregular in space and time at the same scales at which the tracer's motion is irregular.

In this paper we report on our recent results on developing Lagrangian tools to monitor surface transport and mixing in the ocean. We propose with this aim new Lagrangian criteria that enable to track and quantify the water exchange processes and reveal the underlying physical mechanisms. As an output, we compute different Lagrangian synoptic maps of the regions under study for a given period of year and analyze them. The methos is illustrated with a comparatively small marine bay, the Peter the Great Bay in the Japan Sea near Vladivostok (Russia), and a comparatively large region in the North Pacific, the Kuroshio Extension system. In the first case we have used velocity data from a Japan Sea eddy-resolved circulation numerical model with the fine resolution of $2.5 \mathrm{~km}$, in the second one - satellite altimetric velocity data with the coarse resolution of the order of $35 \mathrm{~km}$.

\section{Lagrangian and dynamical systems meth- ods to study transport and mixing in the ocean}

Motion of a fluid particle in a two-dimensional flow is the trajectory of a dynamical system with given initial conditions governed by the velocity field computed either by solving the corresponding master equations or as the output of a numerical ocean model or derived from a measurement

$$
\frac{d x}{d t}=u(x, y, t), \quad \frac{d y}{d t}=v(x, y, t),
$$

where $(x, y)$ is the location of the particle, $u$ and $v$ are the zonal and meridional components of its velocity. Even if the Eulerian velocity field is fully deterministic, the particle's trajectories may be very complicated and practically unpredictable. It means that a distance between two initially nearby particles grows exponentially in time

$$
\|\delta \mathbf{r}(t)\|=\|\delta \mathbf{r}(0)\| e^{\lambda t}
$$


where $\lambda$ is a positive number, known as the Lyapunov exponent, which characterizes asymptotically the average rate of the particle dispersion, and $\|\cdot\|$ is a norm of the vector $\mathbf{r}=(x, y)$. It immediately follows from (3) that we are unable to forecast the fate of the particles beyond the so-called predictability horizon

$$
T_{p} \simeq \frac{1}{\lambda} \ln \frac{\|\Delta\|}{\|\Delta(0)\|}
$$

where $\|\Delta\|$ is the confidence interval of the particle location and $\|\Delta(0)\|$ is a practically inevitable inaccuracy in specifying the initial location. The deterministic dynamical system (2) with a positive maximal Lyapunov exponent for almost all vectors $\delta \mathbf{r}(0)$ (in the sense of nonzero measure) is called chaotic. It should be stressed that the dependence of the predictability horizon $T_{p}$ on the lack of our knowledge of exact location is logarithmic, i. e., it is much weaker than on the measure of dynamical instability quantified by $\lambda$. Simply speaking, with any reasonable degree of accuracy on specifying initial conditions there is a time interval beyond which the forecast is impossible, and that time may be rather short for chaotic systems.

Since the phase plane of the two-dimensional dynamical system (2) is the physical space for fluid particles, many abstract mathematical objects from dynamical systems theory (stationary points, KAM tori, stable and unstable manifolds, periodic and chaotic orbits, etc.) are material surfaces, curves and points in fluid flows. It is well known that besides "trivial" elliptic fixed points, the motion around which is stable, there are hyperbolic fixed points which organize fluid motion in their neighbourhood in a specific way. In a steady flow the hyperbolic points are typically connected by the separatrices which are their stable and unstable invariant manifolds. In a time-periodic flow the hyperbolic points are replaced by the corresponding hyperbolic trajectories with associated invariant manifolds which in general intersect transversally resulting in a complex manifold structure known as a heteroclinic tangle. The fluid motion in these regions is so complicated that it may be strictly called chaotic, the phenomenon known as chaotic advection [8, 5. Adjacent fluid particles in such tangles rapidly diverge providing very effective mechanism for mixing.

Stable and unstable manifolds are important organizing structures in the flow because they attract and repel fluid particles (not belonging to them) at an exponential rate and partition the flow into regions with different types of motion. Invariant manifold in a two-dimensional flow is a material line, i. e., it is composed of the same fluid particles in course of time. By definition 
stable $\left(W_{s}\right)$ and unstable $\left(W_{u}\right)$ manifolds of a hyperbolic trajectory $\gamma(t)$ are material lines consisting of a set of points through which at time moment $t$ pass trajectories asymptotical to $\gamma(t)$ at $t \rightarrow \infty\left(W_{s}\right)$ and $t \rightarrow-\infty\left(W_{u}\right)$. They are complicated curves infinite in time and space that act as boundaries to fluid transport.

The real oceanic flows are not, of course, strictly time-periodic. However, in aperiodic flows there exist under some mild conditions hyperbolic points and trajectories of a transient nature. In aperiodic flows it is possible to identify aperiodically moving hyperbolic points with stable and unstable effective manifolds [4, 3]. Unlike the manifolds in steady and periodic flows, defined in the infinite time limit, the "effective" manifolds of aperiodic hyperbolic trajectories have a finite lifetime. The point is that they play the same role in organizing oceanic flows as do invariant manifolds in simpler flows. The effective manifolds in course of their life undergo stretching and folding at progressively small scales and intersect each other in the homoclinic points in the vicinity of which fluid particles move chaotically. Trajectories of initially close fluid particles diverge rapidly in these regions, and particles from other regions appear there. It is the mechanism for effective transport and mixing of water masses in the ocean. Moreover, stable and unstable effective manifolds constitute Lagrangian transport barriers between different regions because they are material invariant curves that cannot be crossed by purely advective processes.

The stable and unstable manifolds of influencial hyperbolic trajectories are so important because (1) they form a kind of a sceleton in oceanic flows, (2) they divide a flow in dynamically different regions, (3) they are in charge of forming an inhomogeneous mixing with spirals, filaments and intrusions, (4) they are transport barriers separating water masses with different characteristics. Stable manifolds act as repellers for surrounding waters but unstable ones are attractors. That is why unstable manifolds may be rich in nutrients being oceanic "dining rooms".

There is a quantity, the finite-time Lyapunov exponents (FTLE), that enables to detect and visualize stable and unstable manifolds in complex velocity fields. The FTLE is the finite-time average of the maximal separation rate for a pair of neighbouring advected particles which is given by [9]

$$
\lambda(\mathbf{r}(t)) \equiv \frac{1}{\tau} \ln \sigma(G(t)),
$$

where $\tau$ is an integration time, $\sigma(G(t))$ the largest singular value of the evo- 
lution matrix for linearized advection equations. Scalar field of the FTLE is Eulerian but the very quantity is a Lagrangian one that measures an integrated separation between trajectories. Ridges (curves of the local maxima) of the FTLE field visualize stable manifolds when integrating advection equations forward in time and unstable ones when integrating them backward in time.

\section{Transport and mixing in marine bays}

When studing transport and mixing in marine bays, it is important to know which waters enter the bay under study, which ones quit the bay, by which transport corridors they do that and how the different waters mix in the bay interior. The Lagrangian approach, allowing to compute the origin and fate of different waters, is the most suitable for that. Transport and mixing in marine bays is more inhomogeneous as compared with those processes in open basins because of a complicated structure of currents and eddies of different scales, strong tides and presence of river estuaries. In this section we apply Largangian tools to characrerize horizontal subsurface transport and mixing in the Peter the Great Bay near Vladivostok city (Russia). That is the largest bay in the Japan Sea with a few shallow-water smaller bays and estuaries of three major rivers with a wide shelf and steep continental slope. The water exchange between the bay and the open sea is governed mainly by a cyclonic circulation over the deep central basin and the Primorskoye current flowing to the southwest along the continental slope of the Primorsky Krai (Russia). We have used velocity data from the MHI ocean circulation model [10] which is a set of $3 \mathrm{D}$ primitive equations in $Z$-coordinate system with 10 quasi-isopycnal layers and the resolution of $2.5 \mathrm{~km}$.

To characterize the water exchange between the Peter the Great Bay and the open sea we compute the FTLE map and the exit-time map (Fig. 1). A large number of synthetic particles have been uniformly distributed over the region with $\left[130^{\circ} 12^{\prime}: 133^{\circ} 12^{\prime}\right] \mathrm{E}$ and $\left[41^{\circ} 42^{\prime}: 43^{\circ} 19^{\prime}\right]$ N. In Fig. 17a we compute the FTLE, $\lambda$, by the method proposed in Ref. [9]. The advection equations (2) have been integrated forward in time for 54 days in the August and September of a typical year. The gray shades code the magnitude of $\lambda$. The value $\lambda=0.085$, at which the distance between neighbouring particles increases in 100 times, is chosed to be a threshold. The regions with $\lambda<0.085$ are supposed to be regular, the ones with $\lambda>0.085$ - chaotic. The black 
ridges with $\lambda \gg 0.085$ visualize stable manifolds of influencial hyperbolic trajectories in the region. Spiral-like structures reveal eddies of different scales, the white and light-grey zones are the stagnation regions or shear currents. The sandwich-like structures are signs of the most intense mixing. The synoptic Lyapunov map in Fig. 1a shows the scalar filed of this quantity in geographic coordinates which are initial positions of the synthetic particles. This map along with the Lyapunov map, computed backward in time (not shown), demonstrates with a high resolution the complicated character of transport and mixing in the Peter the Great Bay.

The exit-time map is shown in Fig. 1b. The color in the map codes the time, $T$, particles (initially distributed over the same region) need to reach the open sea or the coastline. In fact, we compute the trajectories till they reach the $3 \mathrm{~km}$ band along the coastline. The white wide band along the coast in Fig. 1b demonstrates the Primorskoye current along which particles quickly leave the bay to the southwest. The large white corridor in the central part of the region selected separates the Peter the Great Bay from the open sea. Black color marks the particles that did not leave the bay for the computation time, 54 days. The stagnation zones are situted, as expected, in the smaller bays, the Amursky and the Ussyrisky ones, which are visible as black spots on the both sides of the peninsula in the north. The exit-time map reveals the complicated process of chaotic mixing in the central part of the bay with the spiral-like anticyclonic eddy (with the center at $132^{\circ} 45^{\prime} \mathrm{E}$ and $42^{\circ} 40^{\prime} \mathrm{E}$ ) and gives a valuable information about origin and fate of waters.

To get an information about the character of motion of different waters, their drift, rotation and oscillation, we compute the new Lagrangian synoptic maps: rotation and mixing maps, transport and visitor maps. We compute for a large number of particles the number of cyclonic, $\eta_{c}$, and anticyclonic, $\eta_{a}$, rotations and their difference $\eta$. The typical kinds of particle's motion are the following: 1) simple drift or linear displacement if $\eta_{c}, \eta_{a},|\eta|<\eta_{\mathrm{cr}}=5$, where $\eta_{\mathrm{cr}}$ is a threshold value of the rotation number; 2) rotation, if $|\eta|>\eta_{\mathrm{cr}}=5$; 3) oscillation, if $\eta_{c}, \eta_{a}>\eta_{\mathrm{cr}}=5$ but $|\eta|<\eta_{\mathrm{cr}}$. In the rotation map in Fig. 2 a white and black colors mean cyclonic, $\eta_{c}$, and anticyclonic, $\eta_{a}$, rotations, respectively, computed for the same period of time, 54 days. Grey color codes the particle with predominant displacements or oscillations. The map demonstrates clearly the same spiral-like anticyclonic eddy as in Fig. 1 and the large-scale filaments with foldings typical to chaotic advection in the ocean. 

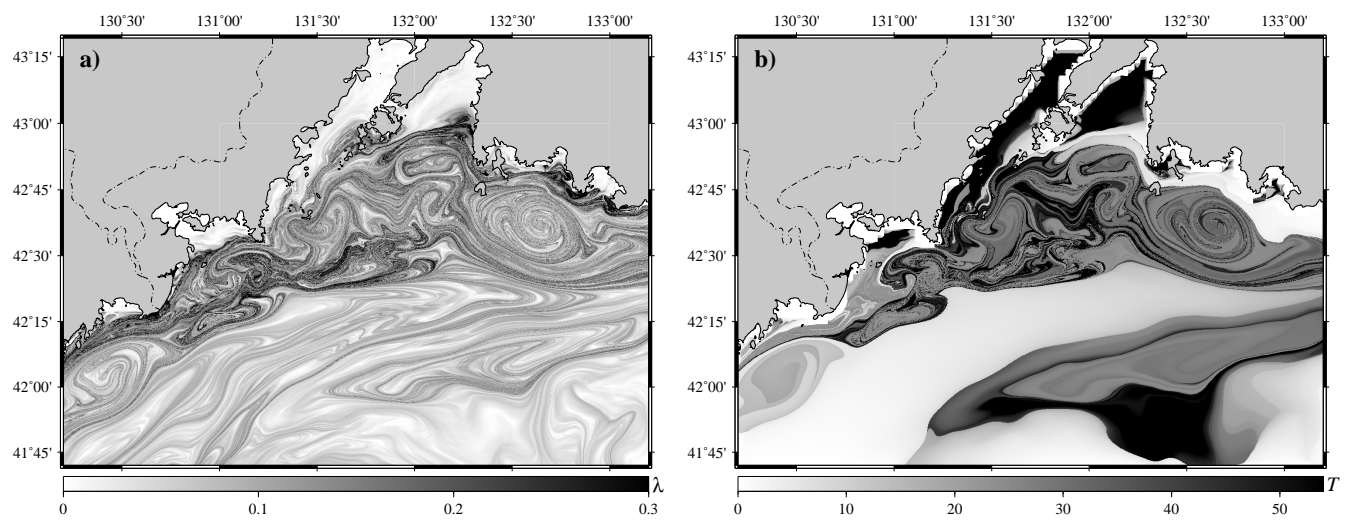

Figure 1: (a) The Lyapunov map in the Peter the Great Bay and the surrounding region of the Japan Sea. (b) The exit-time map in the same region.

To characterize the chaotic mixing more clearly we compute along with the rotation numbers the FTLE $\lambda$. If $\lambda>\lambda_{\text {cr }}=0.85$ and $\eta_{a}>\eta_{\text {cr }}=5$, we will speak about unstable rotations in the corresponding region. If $\lambda>\lambda_{\mathrm{cr}}=0.85$ but $\eta_{a}<\eta_{\text {cr }}=5$ one deals with unstable linear displacement of the corresponding particles. The mixing map in Fig. 2 b shows by color regions with different dynamical properties specified by the rotation numbers and the maximal Lyapunov exponent. White color marks the regions with regular oscillations and/or predominant displacements. The spots of particles, placed in those regions, move as whole being deformed slightly. The white grey color - the regions with unstable displacements which are peripheries of the anticyclonic eddies and their filaments. The spots, placed in those regions, are elongated strongly. The dark grey color — the regions with unstable oscillator motion with the particles rotating for 54 days in the cyclonic and then in the anticyclonic directions. The black color corresponds to the unstable rotation that manifests itself in narrow filaments and spiral-like structures in anticyclones.

In order to find frontal zones and transport pathways we propose to compute the transport maps showing the final positions of particles when integrating the advection equations (2) forward and backward in time (see Figs. 3a and 3b, respectively). In other words, the equations (2) have been solved for each of the million particles initially distributed over the region selected for 54 days forward and backward in time. In the first case we get 

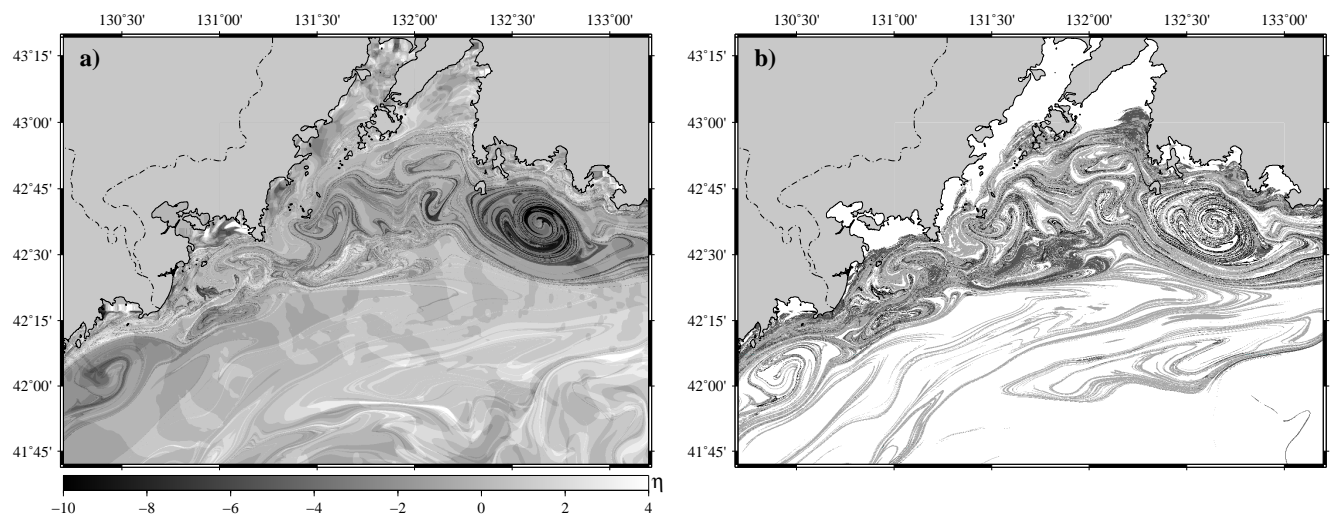

Figure 2: (a) Rotation and (b) mixing maps in the Peter the Great Bay.

the particle's fate map (Fig. 3a) with the black (white) particles leaving the bay through the eastern (western) border. The grey particles are those that did not leave the bay for the computation time. When integrating the equations (2) backward in time, we get the particle's origin map with the black (white) particles entering the bay through the eastern (western) border and the resident particles shown in grey. The frontal zone, separating the waters with different fate and origin, consistes of smooth, meandered and spiral-like fragments.
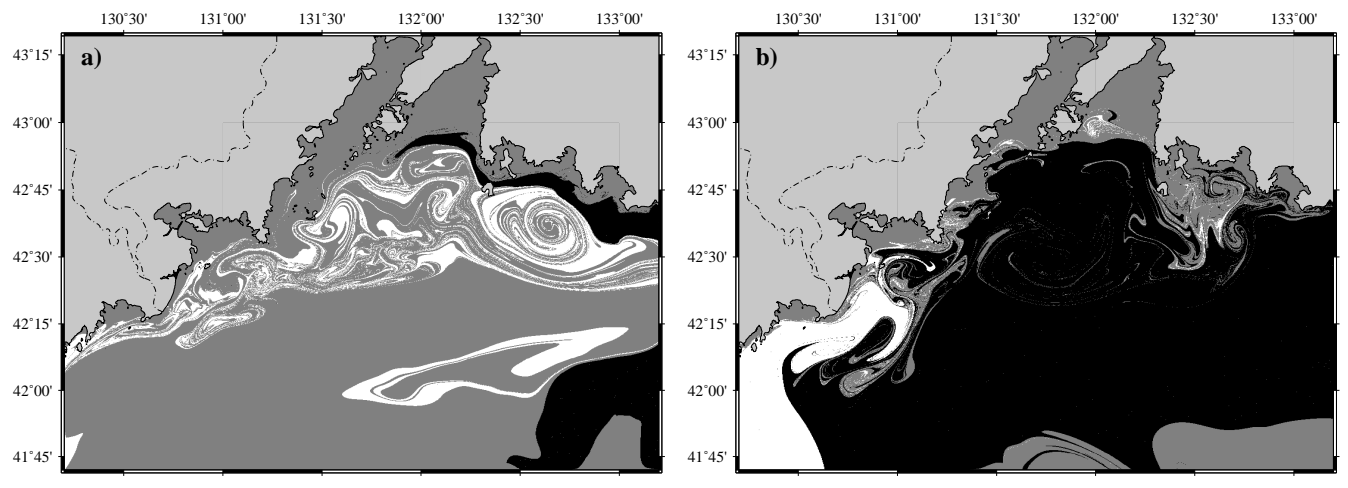

Figure 3: Transport maps in the Peter the Great Bay. (a) The particle's fate and (b) origin maps. 


\section{Transport and mixing in the Kuroshio Ex- tension region}

The Kuroshio Extension prolongs the Kuroshio Current when the latter separates from the continental shelf at about $30^{\circ} \mathrm{N}$. It flows eastward from this point as a strong unstable meandering jet constituting a front separating the warm subtropical and cold subpolar waters of the North Pacific Ocean. It is a region with one of the most intense air-sea heat exchange and the highest eddy kinetic energy level strongly affecting climate. Transport of water masses is of cruicial importance and may cause heating and freshing of waters with a great impact on the weather and living organisms.

The surface ocean currents used in this section are derived from satellite altimeter measurements of sea height (http://www.aviso.oceanobs.com). The velocity data covers the period from 1992 to 2011 with weekly data on a $1 / 3^{\circ}$ Mercator grid. In our study we focus on the region between $30^{\circ}$ and $45^{\circ} \mathrm{N}$ and between $130^{\circ}$ and $165^{\circ}$ E. Bicubical spatial interpolation and third order Lagrangian polinomials in time have been used to provide accurate numerical results. Lagrangian synoptic maps, manifolds and chaotic advection structures in general are determined by the large-scale advection field, which is appropriately captured by altimetry. Thus, computation of particle's trajectories statistically is not especially sensitive to imperfections of the velocity field caused by the interpolation and measurement imperfections.

In Fig. 4 a we demonstrate the displacement map for the region computed for 45 days after the beginning of the incident at the Fukushima Daiichi nuclear power plant. The shades of gray depict the magnitude of the displacement of a tracer $D=\sqrt{\left(x_{f}-x_{0}\right)^{2}+\left(y_{f}-y_{0}\right)^{2}}$, from its initial position, $\left(x_{0}, y_{0}\right)$, to a final one $\left(x_{f}, y_{f}\right)$. The Kuroshio Current is well pronounced including meanders and intrusions, its extension, and mesoscale eddies. Two light-colored eddy patches are of particular interest. Their centers are approximately at the latitude of the Fukushima plant and at longitudes $153^{\circ} \mathrm{E}$ (the mushroom-like dipolar eddy) and $161^{\circ} \mathrm{E}$ (the circular eddy), both eddies being surrounded by dark-colored necklaces having a relatively high magnitude of $D$. This pattern exemplifies the ring birth process due to the meandering of the Kuroshio current and subsequent detachment of eddies from the main jet.

To get a picture of an "instantaneous " state of the region we show in Fig. 4b the surface velocity field computed on the fixed day, 1 January, 2010. 

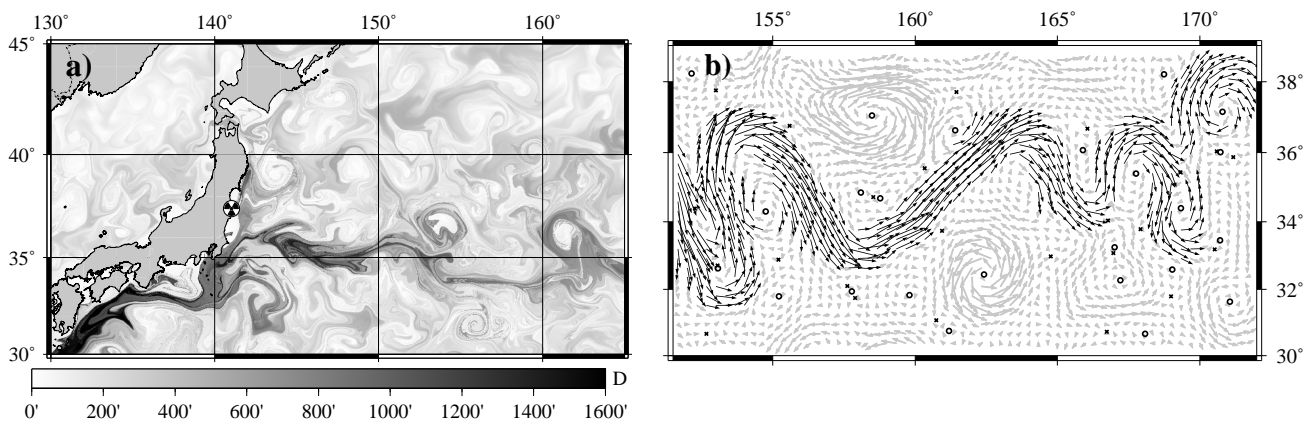

Figure 4: (a) Displacement map in the Kuroshio Extension region. The color codes the magnitude of displacement $D$ in minutes. The Fukushima Daiichi nuclear power plant is marked with the sign of radioactivity. (b) Velocity field of the region on 1 January, 2010. Circles and crosses are instantaneous elliptic and hyperbolic points, respectively.

The main meandering jet is depicted by the black arrows corresponding to comparatively large velocities. The grey arrows around the jet with smaller velocities reveal a number of cyclonic and anticyclonic eddies on both flanks of the jet. We have computed instantaneous elliptic and hyperbolic points of the flow and showed them by circles and crosses, respectively. The elliptic points are situated mainly in the centers of the eddies, whereas the hyperbolic ones are in the regions between the eddies with different polarity and in the periphery of isolated eddies. The hyperbolic points are especially important because they may be connected by instantaneous stable and unstable manifolds dividing the flow into regions with cardinally different dynamics.

We plan to show in this section that the Lagrangian diagnostics is well suitable to describe the mesoscale and submesoclace features of the complex picture of mixing in the Kuroshio Extension region. The altimetric velocity data we used covers the period from 1 January to 3 July, 2010. We focus on a vortex pair on the jet's southern flank, consisting of the anticyclone (AC) and cyclone $(\mathrm{C})$. The pair manifests itself on the Lagrangian maps in Fig. 5 computed for a large number of synthetic particles seeded over the region considered. All the maps visualize the northern hat-like $\mathrm{AC}$ with the axes of 150 and $100 \mathrm{~km}$ and the southern circular $\mathrm{C}$ with the diameter $150 \mathrm{~km}$. In Fig. 5 a the color codes the meridional displacement, $D_{y}$, of particles on the 60th day of integration. The spiral structure of the $\mathrm{C}$ is well developed 
with the spiral untwisting counter-clockwise, whereas it is less pronounced for the $\mathrm{AC}$ with the spiral untwisting clockwise. The character of the water motion in the $\mathrm{C}$ and $\mathrm{AC}$ is also different and becomes evident after computing the number of particle's rotation around the vortex centers. It follows from Fig. 5 b that water in the $\mathrm{AC}$ core circulates with approximately the same angular velocity, whereas this quantity decreases from the center of the $\mathrm{C}$ to its periphery (pay attention to the ring-like structure of the $\mathrm{C}$ ). In order to visualize the stable manifolds of the hyperbolic trajectories around the vortex pair, we compute in Fig. 55c the FTLE, $\lambda$, and displacements, $D$, of the particles. The shades of grey in this figure modulate different combinations and magnitudes of $\lambda$ and $D$ with respect to some chosen "critical" values: $\lambda_{\mathrm{cr}}$, corresponding to divergence of initially close particles over $100 \mathrm{~km}$, and $D_{\text {cr }}=100 \mathrm{~km}$. The black convoluted curves in the figure between the eddies, around each of them and around the very pair delineate the corresponding $W_{s}$ manifolds.
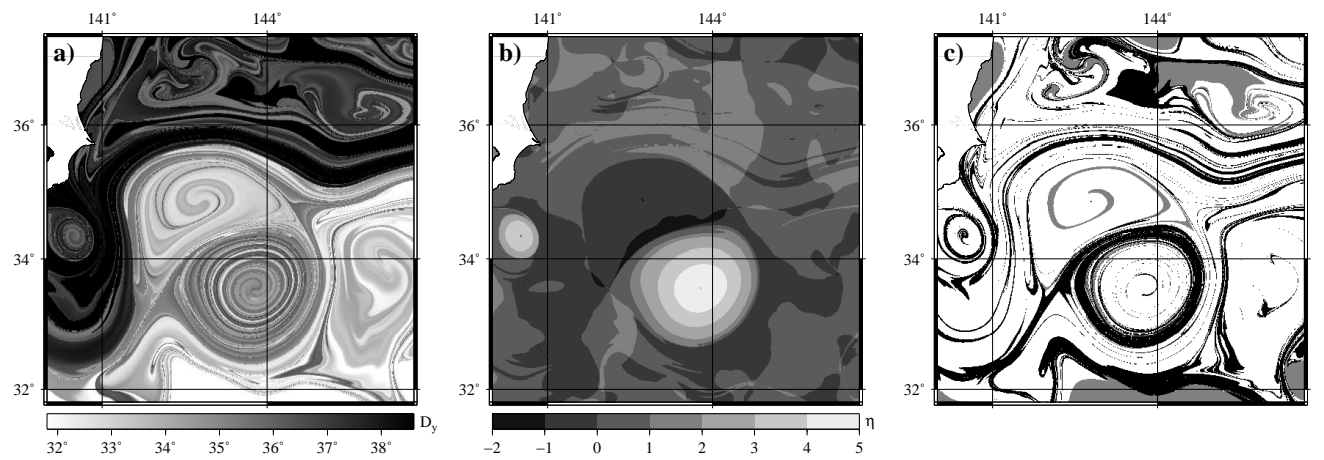

Figure 5: Lagrangian maps of the $\mathrm{AC}-\mathrm{C}$ vortex pair in which the color codes: (a) the meridional displacement of synthetic particles, $D_{y}$, on the 60 th day of integration, (b) the number of their rotation around the vortex centers on the 15th day and (c) their Lyapunov exponents, $\lambda$, and, $D$, displacements on the 45th day with the following legenda: white means $\lambda<\lambda_{\mathrm{cr}}, D \geq D_{\mathrm{cr}}$, grey $-\lambda<\lambda_{\text {cr }}, D<D_{\text {cr }}$ and black $-\lambda \geq \lambda_{\text {cr }}, D \geq D_{\text {cr }}$.

To give a detailed description of the structure of each eddy in the vortex pair we apply the method of particle's scattering elaborated in Ref. [1. We cross both the eddies by a material line and compute rotation number $\eta$, and the maximal FTLE on initial particle's latitude $y_{0}$. The scattering plot in 
Fig. 6a demonstrates that the waters in the $\mathrm{C}$ core really rotate with different angular velocities decreasing from the center to its periphery. Rotation in the $\mathrm{AC}$ core is much more homogeneous. Moreover, the waters in the $\mathrm{C}$ rotate in two times faster than in the AC. The scattering plot $\lambda\left(y_{0}\right)$ in Fig. $6 \mathrm{~b}$ demonstrates smooth segments in the cores of $\mathrm{C}$ and $\mathrm{AC}$ and irregular oscillations in their periphery. It simply means that the water in the cores moves more or less coherently whereas the motion in the eddy's peripheries is erratic due to numerous intersections of stable and unstable manifolds. Computation of the dependence of the time of exit of the particles $T$, belonging to the material line, on $y_{0}$ (not shown) confirms that waters prefer to quit the $\mathrm{C}$ more or less periodically by portions. Each portion is represented by a $\cup$-like segment of the $T\left(y_{0}\right)$ function which consists of a large number of particles with approximately the same time of exit and the same rotation number $\eta$. In difference from the $\mathrm{C}$, particles quit the $\mathrm{AC}$ core practically at the same time. In other words, the particles quit the $\mathrm{C}$ by portions along spiral-like transport pathways, whereas the periphery of the $\mathrm{AC}$ exchanges water with the surrounding but its core moves coherently as a whole for a time.
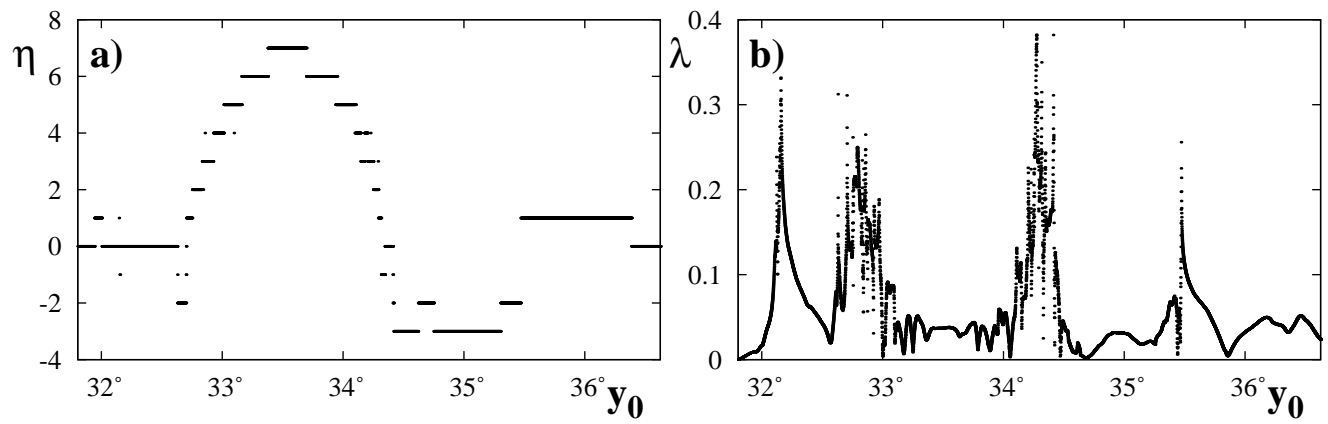

Figure 6: The scattering plots for the vortex pair on the 30th day of integration. (a) Number of times, $\eta$, the particles rotate around the vortex centers vs initial particle's latitude position $y_{0}$, (b) the corresponding maximal FTLE vs $y_{0}$.

In conclusion we demonstrate in Fig. 7 how frequently fluid particles, chosen in the cores of the $\mathrm{C}$ and $\mathrm{AC}$, visit for 180 days different places in the Kuroshio Extension region. It is evident that the $\mathrm{C}$ was absorbed by the main jet in a short time and then its waters travelled eratically within the jet with a few excursions to its northern and southern flanks. It is 
interesting that in course of time $\mathrm{C}$ waters have formed the new cyclonic eddy nearby $\left(x_{0}=155^{\circ} \mathrm{E}, y_{0}=32^{\circ} \mathrm{N}\right)$. In contrast to the $\mathrm{C}$, the $\mathrm{AC}$ waters have walked eratically on the southern flank of the jet in a restricted region within $x_{0}=\left[140^{\circ}: 150^{\circ}\right] \mathrm{E}, y_{0}=\left[28^{\circ}: 35^{\circ}\right] \mathrm{N}$.

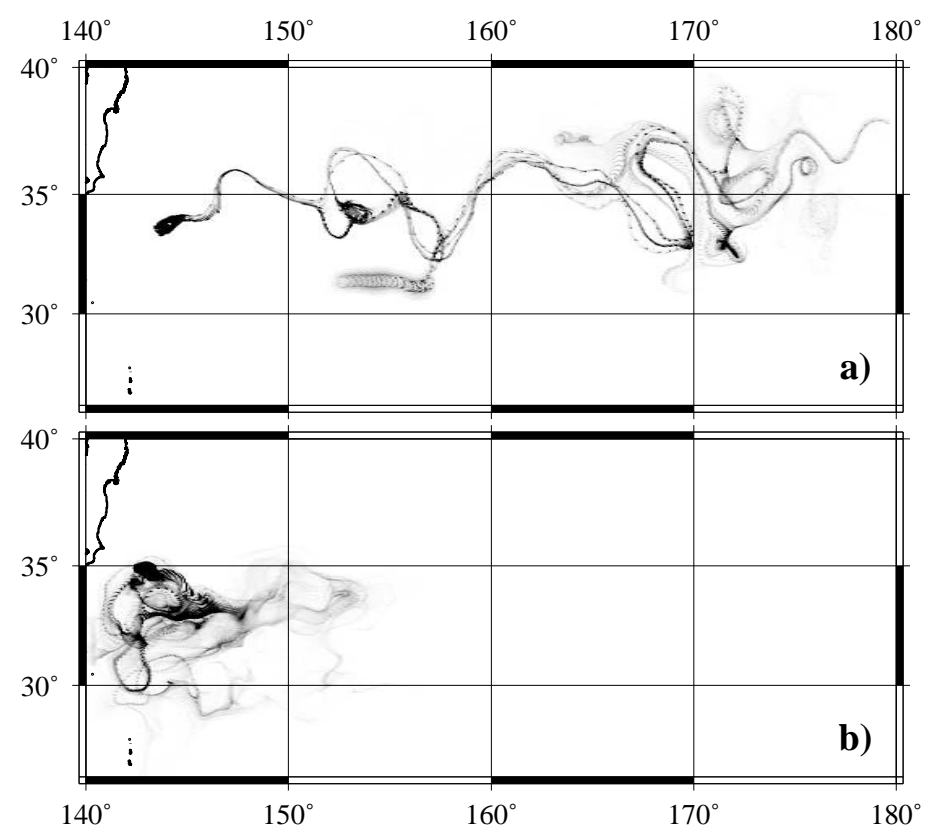

Figure 7: Visitor maps for (a) the cyclone and (b) anticyclone show how frequently fluid particles from the corresponding eddy's cores visit for 180 days different places in the Kuroshio Extension region.

\section{Conclusion}

The Lagrangian approach has been shown to be very useful to gain new information on chaotic transport and mixing in the ocean. We have elaborated new Lagrangian diagnostic tools to visualize and quantify those processes: the time of exit of fluid particles off a selected box, their displacements, the number of their cyclonic and anticyclonic rotations and the number of times they visit different places in the region. Along with the Lyapunov maps, the corresponding high-resolution Lagrangian synoptic maps of those 
quantities, computed by solving advection equations forward and backward in time for different periods of the year, are new diagnostic and prognostic products characterizing the state of the ocean. The technique developed can be applied to the global ocean and its basins.

In this paper we have focused on a comparatively small marine bay, the Peter the Great Bay in the Japan Sea near Vladivostok (Russia), and on a comparatively large region in the North Pacific, the Kuroshio Extension system. In the bay study in summer and autumn periods, we have used the velocity data from a Japan Sea eddy-resolved circulation numerical model with the resolution of $2.5 \mathrm{~km}$. It has been shown that the Lyapunov and exit-time maps, the rotation, mixing and transport maps allowed to quantify and specify movement of water masses, their mixing and the degree of its chaoticity in the bay. Those high-resolution maps allowed to visualize transport pathways by which waters exit and enter the bay.

As to the Kuroshio Extension, we have used the velocity data derived from satellite altimeter measurements of sea height with the corresponding interpolation. The main attention has been paid to study structure, transport and mixing of a vortex pair with strongly interacting cyclonic and anticyclonic eddies. Such dipoles occur frequently in that region. We have computed Lagrangian synoptic maps for the time of exit of particles, the number of changes of the sign of zonal and meridional velocities, and for other quantities. Along with the Lyapunov map, they have been shown to be able to reveal the vortex structure and its evolution, meso- and submesoscale filaments, repelling material lines, hyperbolic and non-hyperbolic regions in the sea. In particular, we have found that the eddies have a prominent spiral-like structure resembling the spiral patterns at satellite images in that region.

The work was supported by the Program "Fundamental Problems of Nonlinear Dynamics" of the Russian Academy of Sciences, by the Russian Foun-

dation for Basic Research (projects nos. 09-05-98520 and 11-01-12057) and by the Prezidium of the Far-Eastern Branch of the RAS.

\section{References}

[1] M. V. Budyansky, M. Yu. Uleysky, and S. V. Prants, JETP 99 (2004), no. 5, 1018-1027. 
[2] Francesco d'Ovidio, Jordi Isern-Fontanet, Cristóbal López, Emilio Hernández-García, and Emilio García-Ladona, Deep Sea Research Part I: Oceanographic Research Papers 56 (2009), no. 1, 15-31.

[3] G. Haller, Physics of Fluids 14 (2002), no. 6, 1851-1861.

[4] G. Haller and A.C. Poje, Physica D: Nonlinear Phenomena 119 (1998), no. 3-4, 352-380.

[5] K.V. Koshel and S.V. Prants, Physics Uspekhi 49 (2006), 1151-1178.

[6] Francois Lekien, Chad Coulliette, Arthur J. Mariano, Edward H. Ryan, Lynn K. Shay, George Haller, and Jerry Marsden, Physica D: Nonlinear Phenomena 210 (2005), no. 1-2, 1-20.

[7] Ana M. Mancho, Des Small, and Stephen Wiggins, Physics Reports 437 (2006), no. 3-4, 55-124.

[8] J.M. Ottino, The kinematics of mixing: Stretching, chaos, and transport, Cambridge University Press, Cambridge, U.K., 1989.

[9] S. V. Prants, M. V. Budyansky, V. I. Ponomarev, and M. Yu. Uleysky, Ocean modelling 38 (2011), no. 1-2, 114-125.

[10] S. V. Prants, V. I. Ponomarev, M. V. Budyansky, M. Yu. Uleysky, and P. A. Fayman, Izvestiya, Atmospheric and Oceanic Physics (in press).

[11] Emilie Tew Kai, Vincent Rossi, Joel Sudre, Henri Weimerskirch, Cristobal Lopez, Emilio Hernandez-Garcia, Francis Marsac, and Veronique Garçon, PNAS 106 (2009), no. 20, 8245-8250.

[12] Darryn W. Waugh, Edward R. Abraham, and Melissa M. Bowen, Journal of Physical Oceanography 36 (2006), no. 3, 526-542. 